\title{
Nuclear Data for Proton Beam Radiotherapy
}

\author{
Maria Eugênia de Melo Rêgo a and B. V. Carlson ${ }^{\mathrm{b}}$ \\ ${ }^{a}$ Univ. Est. Paulista "Julio de Mesquita Filho" - UNESP - campus Guaratinguetá \\ Av. Dr. Ariberto Pereira da Cunha, 333 - 12500-000-Guaratinguetá, São Paulo-Brasil \\ ${ }^{b}$ Instituto Tecnológico de Aeronáutica-ITA \\ Pça Marechal Eduardo Gomes, 50 - 12228-900 - São José dos Campos, São Paulo-Brasil
}

\begin{abstract}
The need for a program of work focused on the nuclear data evaluation of chargedparticle reactions has arisen recently due to their increasing use in cancer therapy. This project, as part of that program, has as its main goal the selection and comparison of nuclear data for nuclear reactions induced by protons at low to intermediate energies $(\mathrm{E}<250 \mathrm{MeV})$. The methodology of selection was based on the data base EXFOR and the compilations of radionuclide production cross sections of N. Sobolevsky. For the purpose of comparison and evaluation, theoretical calculations with the reaction model code EMPIRE-II are being used.
\end{abstract}

Keywords: EMPIRE-II, EXFOR, nuclear, proton, radiotherapy.

PACS: N 29.85.-c, N 29.85.Fj

\section{Introduction}

Proton beam therapy has been confirmed as an efficacious means for the radiation treatment of tumors, which minimizes damages caused to nearby healthy tissues as well.

This therapy was proposed by Robert Wilson in 1946, who observed that the beam had a nearly straight-line path due to the particles mass and that the bulk of the beam energy was deposited at the maximum range, in a narrow extent about the Bragg peak. This modality of cancer treatment began to be used on patients in 1954 at the Lawrence Berkeley National Laboratory in the USA.

\section{Data selection and analysis}

The EXFOR [1] software and data library provided by the IAEA was used for the selection and extraction of experimental data for reactions involving protons or alpha particles as incident particles, to a maximum energy of $200 \mathrm{MeV}$, with carbon-12, nitrogen-14, oxygen-16, aluminium-27, silicon-28 or iron-56 atoms as targets. After selecting the characteristics desired of the data, this software provides names, reference code, author and publishing location, a short abstract and the available experimental data.

For this project, we chose experiments that furnish a reasonable number of data points for elastic or inelastic differential angular scattering of proton, neutron or alpha

CP1139, Nuclear Physics 2008, XXXI Workshop on Nuclear Physics in Brazil, edited by V. Guimarães, J. R. B. Oliveira, K. C. D. Macario, and F. A. Genezini (C) 2009 American Institute of Physics 978-0-7354-0676-6/09/\$25.00 
emission spectra or double differential angle/energy cross sections. We also extracted related production cross section data from EXFOR and from N. Sobolevsky's compilation [2], for comparison to theoretical model calculations.

After compiling the available data, we began to study the EMPIRE-II system [3], a set of model codes for nuclear reaction calculations. In this system, we select the reaction characteristics and theoretical models to be used for calculations. A huge library of input parameters provides everything from nuclear masses, optical model parameters, ground state deformations and branching ratios for decay to discrete levels.

\section{Results and Discussion}

Tables were prepared in order to facilitate the comparison of the data sets. The total energy range, reaction, reference code, angular range, level energy or energy of outgoing particle and number of data points are indicated in the tables.

Discrepancies exist between experimental and theoretical model results, at both low and high nucleon emission energies. The differences at low energies are due to the fact that compound nucleus emission is not included in the spectra taken from the EMPIRE output, a defect of the code that is being remedied. We are still investigating the discrepancies at higher energies, which have the characteristics of a misplaced quasi-elastic peak.

Up to this point, we have had only partial success with this project, studying experimental methods and nuclear reactions models, collecting and selecting data from EXFOR and preparing tables. Automation of graph preparation will be the next important step in facilitating the systematic use of EMPIRE-II.

Another serious difficulty with the use of EMPIRE-II is that it was designed to calculate neutron-induced cross sections rather than proton-induced ones. As soon as graph preparation is more automatic and problems with low-energy proton-induced reactions are fixed, we plan to perform a systematic study of the compiled data. We plan to use the hybrid Monte Carlo pre-equilibrium model of Blann and Chadwick [4] as a basis for this study.

\section{Acknowledgments}

MEMR acknowledges partial support from the IAEA and the CNPq. BVC acknowledges partial support from the IAEA, the CNPq and from FAPESP.

\section{References}

1. Experimental Nuclear Reaction Data - EXFOR, last access December $4^{\text {th }}, 2008$. www-nds.iaea.org/exfor'exfor00.htm.

2. N. Sobolevsky, 2007, "Cross Sections of Radionuclide Production in Nuclear Reactions in Biological Tissue", INDC (NDS)-0504, IAEA, Áustria.

3. Herman, M., 2002, "EMPIRE-II Statistical Model Code for Nuclear Reaction Calculations: Version 2.17 Millesimo", IAEA, Áustria.

4. M. Blann and M.B. Chadwick, Phys. Rev. C57, 233 (1998). 
Copyright of AIP Conference Proceedings is the property of American Institute of Physics and its content may not be copied or emailed to multiple sites or posted to a listserv without the copyright holder's express written permission. However, users may print, download, or email articles for individual use. 